PERSPECTIVE

\title{
What's in a Label? Is Diagnosis the Start or the End of Clinical Reasoning?
}

\author{
Jonathan S. Ilgen, MD, MCR ${ }^{1,2}$, Kevin W. Eva, $P h D^{3,4}$, and Glenn Regehr, $P h D^{4,5}$
}

'Department of Medicine, Division of Emergency Medicine, University of Washington School of Medicine, Seattle, WA, USA; ${ }^{2}$ Center for Leadership \& Innovation in Medical Education, University of Washington School of Medicine, Seattle, WA, USA; ${ }^{3}$ Department of Medicine, University of British Columbia, Vancouver, British Columbia, Canada; ${ }^{4}$ Centre for Health Education Scholarship, University of British Columbia, Vancouver, British Columbia, Canada; ${ }^{5}$ Department of Surgery, University of British Columbia, Vancouver, British Columbia, Canada.

Diagnostic reasoning has received substantial attention in the literature, yet what we mean by "diagnosis" may vary. Diagnosis can align with assignment of a "label," where a constellation of signs, symptoms, and test results is unified into a solution at a single point in time. This "diagnostic labeling" conceptualization is embodied in our case-based learning curricula, published case reports, and research studies, all of which treat diagnostic accuracy as the primary outcome. However, this conceptualization may oversimplify the richly iterative and evolutionary nature of clinical reasoning in many settings. Diagnosis can also represent a process of guiding one's thoughts by "making meaning" from data that are intrinsically dynamic, experienced idiosyncratically, negotiated among team members, and rich with opportunities for exploration. Thus, there are two complementary constructions of diagnosis: 1) the correct solution resulting from a diagnostic reasoning process, and 2) a dynamic aid to an ongoing clinical reasoning process. This article discusses the importance of recognizing these two conceptualizations of "diagnosis," outlines the unintended consequences of emphasizing diagnostic labeling as the primary goal of clinical reasoning, and suggests how framing diagnosis as an ongoing process of meaningmaking might change how we think about teaching and assessing clinical reasoning.

KEY WORDS: diagnostic reasoning; clinical reasoning; diagnosis; problem-solving; medical decision-making.

$\mathrm{J}$ Gen Intern Med 31(4):435-7

DOI: $10.1007 / \mathrm{s} 11606-016-3592-7$

(c) Society of General Internal Medicine 2016

\section{INTRODUCTION}

"I solved the case, my work is done."

—Dr. Gregory House, "House, M.D.” TV series, Pilot

Episode

Often times in medicine, "making the diagnosis" is treated as the goal of clinical problem solving, tantamount to finding the

Received August 5, 2015

Revised December 9, 2015

Accepted January 7, 2016

Published online January 26, 2016 solution to a riddle. Framed in this way, diagnosis is an act of categorization, culminating in the endpoint of a correct "diagnostic label." This framing of the process and goal of diagnosis is ubiquitous in our cultural references, our journals, and our research studies. Elite diagnosticians, whether identified by peers ${ }^{1}$ or portrayed by the cantankerous Dr. Gregory House on television, are revered for their ability to assemble seemingly disparate pieces of information into unifying diagnostic labels when others have failed. In clinical journals, many case reports artificially pause with a teaser that "a diagnostic test was performed" before engaging the reader in a comprehensive review of the diagnostic possibilities that ultimately culminate in the "correct" answer. Research on clinical expertise, heuristics and biases, and diagnostic error is also largely aligned with this framework, typically using a single unifying diagnosis that is assigned at a single point in time, and measuring diagnostic accuracy as the primary outcome of interest.

It is not surprising that diagnosis has a prominent place in physicians' understanding of their role for (at least) two reasons. First, the sense of satisfaction that comes from identifying a diagnosis can be highly compelling in and of itself, because of the "aha" feeling of having risen to a challenge and figured something out. Second, making a diagnosis can be a powerful aid to clinical reasoning, as it organizes one's thoughts, lessens the load placed on working memory, ${ }^{2}$ and helps to generate explanatory hypotheses for a patient's situation. It is important to note, however, that these two reasons for valuing diagnosis contain critically different framings of the "diagnosis" construct itself: drawing satisfaction from "making a diagnosis" treats diagnosis as a solution (i.e., as the endpoint of a problem solving activity), while using diagnosis as a clinical reasoning aid treats diagnosis as a way of making meaning of the situation in order to better understand and manage a problem. ${ }^{3}$ These two versions of "diagnosis" are not, of course, incompatible. However, the vividness of the first - both in its compelling visceral experience when accomplished and in its celebration in our culture - has the potential of implicitly overvaluing diagnosis as an endpoint in a manner that under-represents the complexity of clinical reasoning. Such overvaluing of diagnostic labeling may exist to some degree in the minds of clinicians, but it almost certainly exists prominently in the minds of our students, and, when it does 
occur, it is likely to have problematic consequences pertaining to humanism, clinical practice, and education.

\section{THE UNINTENDED CONSEQUENCES OF OVEREMPHASIZING DIAGNOSTIC LABELS}

From a humanist perspective, diagnostic labels may oversimplify, or introduce bias into, how clinicians interpret patients' unique illness narratives. By using diagnostic labels as shorthand descriptors for patients, such as "I am going to go see the Crohn's in room 5," the speaker implies a lived experience that is stereotypical and almost certainly devoid of personal intricacies. As described by Mead and colleagues in their conceptual review of patient-centeredness:

A compound leg fracture will not be experienced in the same way by two different patients; it may cause far less distress to the office worker than the professional athlete, for whom the injury potentially signifies the end of a career. Similarly, the medical treatment (even cure) of disease does not necessarily alleviate suffering for all patients., ${ }^{4}$ pg 1089

Labeling patients as their diagnosis undoubtedly impacts how clinicians foster rapport, from difficulties establishing trust with patients who have been labeled as "opiate addicts," to difficulties conveying empathy towards patients with seemingly "simple" problems such as musculoskeletal back pain. In this way, "provider-centered" diagnostic labels can represent an unnecessary barrier to individualized, patient-centered care.

From a practice perspective, diagnostic labeling has ramifications for how interdisciplinary healthcare team members implicitly communicate and interpret clinical data. By distilling a set of symptoms or findings into a diagnostic category, clinicians provide clarity around decision-making and stake their claim regarding what pieces of information have value and what can be considered irrelevant "noise." Yet overemphasis on the diagnostic label also biases team members towards expectations regarding how patients with a particular disease should present clinically ${ }^{4}$ and how they should respond to therapy. ${ }^{5}$ Initial or subsequent symptoms are viewed through this "pre-labeled" prism, and evidence that "does not fit" is often ignored or minimized. ${ }^{6}$ Anchoring in this way is often described as premature closure and implies that "when the diagnosis is made, the thinking stops."7 To avoid complacency with these initial diagnostic labels and remain sensitive to symptom evolution or responses to therapy requires substantial cognitive resources, ${ }^{8}$ as well as team members who are willing to question each others' assumptions and identify pieces of data that may not fit.

From a curricular standpoint, students seem to pick up on our emphasis on diagnostic labeling and treat it as the goal of clinical practice. This may be a result of our own ambiguity in distinguishing between what we mean by "clinical reasoning" and "diagnostic reasoning." When we say clinical reasoning, we are describing a creative and open-ended exploration of a problem that aims to develop an understanding of a situation. In this effort, a diagnosis can be a valuable aid to reasoning, but it does not define the entirety of the reasoning process. In contrast, diagnostic reasoning endeavors to find a unifying label, converging on an answer that is either right or wrong. By emphasizing an approach to practice that privileges diagnostic labels, we should not be surprised when students adopt a performance orientation and "game" case-based learning by trying to get the right answer rather than using the activity as an opportunity to achieve rich conceptual understanding. ${ }^{10}$ Nor should it surprise us when students - implicitly viewing diagnostic labeling as necessary and sufficient for action - seem paralyzed by uncertainty, and frequently rush to place ill-fitting diagnostic labels on ambiguous patient complaints.

\section{IMPLICATIONS OF EMPHASIZING DIAGNOSIS AS "MEANING-MAKING"}

Given the problems inherent in emphasizing diagnosis as the goal of thinking, how might we shift toward more effectively emphasizing the diagnostic process as an aid to thinking, an ongoing exercise of interpretation and "meaning-making?"3 First and foremost, we would privilege curricula that explicitly recognize diagnosis to be dynamic and negotiated. Doing so would involve placing a deliberate emphasis on seeking to understand patients' lived experiences with their illnesses ${ }^{11}$-independent of whether these symptoms can be categorized by traditional disease taxonomies ${ }^{12}$ - and stressing the importance of communication between the healthcare team and patients regarding symptom evolution and responses to therapy. This patient-centered approach has potential to foster empathy and reduce premature closure, given the need to consider multiple perspectives. Second, we would look for instructional designs that emphasize the learning value inherent in complex or ambiguous problems, rooting these experiences in a genuine curiosity concerning how our patients' narratives and physical presentations evolve over time. ${ }^{13,14}$ In doing so, we can be explicit that uncertainty is acceptable and inevitable for all of us, and further, that the act of making decisions or seeking new information provides rich learning opportunities for clinicians and the systems that support them.

Examples of practices that support the dynamic and complex nature of healthcare could include small group learning experiences and assessment activities designed to emphasize the iterative and negotiated nature of clinical care. Preclinical students could be given cases in which the details are sufficiently underspecified, such that no single diagnosis can be definitively determined, or that have branch points with different outcomes depending upon the management decisions the team pursues. At more senior levels of training, a team of residents could be tasked with devising a diagnostic and therapeutic plan for a standardized patient presenting with vague or unusual symptoms. Instead of placing emphasis on 
finding the "correct diagnosis," learners would be encouraged to hypothesize how their patient's symptoms might map to what they know about anatomy and pathophysiology, ${ }^{15}$ model how they search the literature to find clues for what to do, identify whom they might consult for help, and decide upon a safe management plan before a definitive diagnosis is established. Finally, at the bedside with real patients, thinking of diagnosis as 'meaning-making' emphasizes the perpetual importance of curiosity, ${ }^{13}$ not only about symptoms, but about the patients themselves, ${ }^{16}$ in order to avoid both premature closure and a loss of empathy.

Current conceptualizations of adaptive expertise suggest that challenging cases provide clinicians with opportunities for personal growth through collaborating with colleagues, learning independently, or identifying new solutions through innovations in practice. ${ }^{17}$ In this framework, assigning a diagnosis is seen as activating forces for new explorations (what to look for, ${ }^{18}$ what else to expect, ${ }^{19}$ and when to be surprised ${ }^{20}$ ), rather than serving as an endpoint. Thus, "diagnostic success" is no longer framed as a correct or incorrect diagnostic label, but rather is considered a phenomenon that results in an improved (shared) understanding of a problem among healthcare team members and their patients.

\section{SUMMARY}

In sum, there may be at least two ways of understanding what we mean by "diagnosis" in practice: the solution that emerges from the diagnostic problem solving process (i.e., the end-point of the thinking process), and a dynamic framework that is part of the thinking process (i.e., an aid to thinking). Physicians in practice and even formal organizations may well use both conceptualizations interchangeably, as might be the case in The Institute of Medicine's, most recent addition to their Quality Chasm series, where they state: "Getting the right diagnosis is a key aspect of health care: It provides an explanation of the patient's health problem and informs subsequent health care decisions" (emphases added). ${ }^{21}$ However, there may be dangers in doing so without deliberate consideration of how this impacts their clinical reasoning, the interactions between healthcare team members and with patients, how we think about patients, and how we design curricula to support the skills and attitudes of our trainees. For experienced physicians, the ramifications primarily relate to preconceived assumptions regarding lived experiences with particular illnesses and the trap of premature closure, while for more novice learners, mistaking diagnosis as "the answer to a problem" interferes with their ability to tolerate the uncertainty that pervades medical practice and runs the risk of empathy loss. Invoking a desire to "get the answer right" among our trainees likely shortchanges their opportunities for exploration and learning, ${ }^{10}$ and may lead to maladaptive behaviors in their growth toward expert, caring physicians.

\section{Acknowledgements:}

Contributors: We thank the following colleagues for their constructive critiques and insights on this manuscript: Judith Bowen, MD, Professor of Medicine, Oregon Heath \& Science University; Gurpreet Dhaliwal, $M D$, Professor of Medicine, University of California, San Francisco; and Joshua Jauregui, MD, Acting Instructor, University of Washington. No compensation was received for these contributions.

\section{Prior Presentations: None.}

Corresponding Author: Jonathan S. Ilgen, MD, MCR; Department of Medicine, Division of Emergency Medicine, University of Washington School of Medicine, Seattle, WA, USA (e-mail: ilgen@uw.edu).

\section{Compliance with Ethical Standards:}

Conflict of Interest: The authors declare that they do not have a conflict of interest.

Funders: This work was supported by an internal grant from the University of Washington's Center for Leadership \& Innovation in Medical Education (CLIME).

\section{REFERENCES}

1. Eva KW, Lohfeld L, Dhaliwal G, Mylopoulos M, Cook DA, Norman GR. Modern conceptions of elite medical practice among internal medicine faculty members. Acad Med. 2011;86:S50-4.

2. van Merrienboer JJ, Sweller J. Cognitive load theory in health professional education: design principles and strategies. Med Educ. 2010;44:85-93.

3. Bruner JS. Acts of Meaning. Cambridge: Harvard University Press; 1990.

4. Brooks LR, LeBlanc VR, Norman GR. On the difficulty of noticing obvious features in patient appearance. Psychol Sci. 2000;11:112-7.

5. Ortendahl M, Fries JF. Discounting and risk characteristics in clinical decision-making. Med Sci Monit. 2006;12:RA41-5.

6. Wallsten TS. Physician and medical student bias in evaluating diagnostic information. Med Decis Making. 1981;1:145-64.

7. Croskerry P. The importance of cognitive errors in diagnosis and strategies to minimize them. Acad Med. 2003;78:775-80.

8. Epstein RM, Siegel DJ, Silberman J. Self-monitoring in clinical practice: a challenge for medical educators. J Contin Educ Health Prof. 2008;28:5-13.

9. Norman G. Research in clinical reasoning: past history and current trends. Med Educ. 2005;39:418-27.

10. Teunissen PW, Bok HG. Believing is seeing: how people's beliefs influence goals, emotions and behaviour. Med Educ. 2013;47:1064-72.

11. Kleinman A, Eisenberg L, Good B. Culture, illness, and care: clinical lessons from anthropologic and cross-cultural research. Ann Intern Med. 1978;88:251-8.

12. Mead N, Bower P. Patient-centredness: a conceptual framework and review of the empirical literature. Soc Sci Med. 2000;51:1087-110.

13. Schattner A. Curiosity. Are you curious enough to read on? J R Soc Med. 2015;108:160-4.

14. Benbassat J, Baumal R, Heyman SN, Brezis M. Viewpoint: suggestions for a shift in teaching clinical skills to medical students: the reflective clinical examination. Acad Med. 2005;80:1121-6.

15. Woods NN. Science is fundamental: the role of biomedical knowledge in clinical reasoning. Med Educ. 2007;41:1173-7.

16. Fitzgerald FT. Curiosity. Ann Intern Med. 1999;130:70-2

17. Mylopoulos M, Regehr G. How student models of expertise and innovation impact the development of adaptive expertise in medicine. Med Educ. 2009;43:127-32.

18. Baghdady MT, Pharoah MJ, Regehr G, Lam EW, Woods NN. The role of basic sciences in diagnostic oral radiology. J Dent Educ. 2009;73:1187-93.

19. Woods NN, Brooks LR, Norman GR. It all makes sense: biomedical knowledge, causal connections and memory in the novice diagnostician. Adv Health Sci Educ Theory Pract. 2007; 12:405-15.

20. Moulton CA, Regehr G, Mylopoulos M, MacRae HM. Slowing down when you should: a new model of expert judgment. Acad Med. 2007;82:S109-16.

21. National Academies of Sciences, Engineering, and Medicine. Improving diagnosis in health care. 2015; http://www.nap.edu/read/21794/. Accessed November 10, 2015. 